\title{
CORRESPONDENCE OPEN \\ Serum IgA, IgM, and IgG responses in COVID-19
}

\author{
Huan $\mathrm{Ma}^{1,2}$, Weihong Zeng ${ }^{1,2}$, Hongliang $\mathrm{He}^{3}$, Dan Zhao ${ }^{2}$, Dehua Jiang ${ }^{4}$, Peigen Zhou $\mathbb{C}^{5}$, Linzhao Cheng ${ }^{6,7}$, Yajuan $\mathrm{Li}^{8}$, \\ Xiaoling $\mathrm{Ma}^{9}$ and Tengchuan Jin $\mathbb{1}^{6}$
}

Cellular \& Molecular Immunology (2020) 17:773-775; https://doi.org/10.1038/s41423-020-0474-z

Currently, detecting SARS-CoV-2 RNAs is a standard approach for COVID-19 diagnosis. However, there is an urgent need for reliable and rapid serological diagnostic methods to screen SARS-CoV-2infected people including those who do not have overt symptoms. Most emerging studies described serological tests based on detection of SARS-CoV-2-specific $\operatorname{lgM}$ and $\operatorname{lgG} .^{1-4}$ Although detection of SARS-CoV-2-specific IgA in serum has been reported in few papers, ${ }^{5,6}$ analyses of IgA levels in a larger number of COVID-19 patients are still lacking.

This study enrolled a total of 87 confirmed COVID-19 patients (Supplementary Table 1) who were admitted to the First Affiliated Hospital of USTC Hospital or the First Affiliated Hospital of Anhui Medical University between January 26, and Mar 5, 2020. Their blood samples were collected during routine clinical testing. All enrolled cases were confirmed with SARS-CoV-2 infection by use of a standard RT-qPCR assay on throat swab samples from the respiratory tract. For all of the enrolled patients, the date of illness onset, clinical classifications of severity, RNA testing results during the hospitalization period, and the personal demographic information were obtained from the clinical records.

Highly purified receptor-binding domain (RBD) of the SARSCoV-2 spike protein (Supplementary Fig. 1) was expressed in human 293F cells and used to make a set of chemical luminescence kits for detecting the presence of RBD-specific $\lg \mathrm{A}$, $\lg \mathrm{M}$, and IgG, respectively. To evaluate the diagnostic power of the these kits, 216 sera from 87 SARS-CoV-2-infected patients and a total of 483 control sera including 330 healthy sera, 138 "interfering" sera of other-type patients and 15 sera from oncesuspected pneumonia cases were tested. The detected signals relative light units (RLU), for each of isotype of the RBD-specific antibodies, were plotted (Fig. 1a-c). The RBD-specific IgA, IgM, and IgG kits showed diagnostic sensitivities of $98.6 \%, 96.8 \%$, and $96.8 \%$, and specificities of $98.1 \%, 92.3 \%$, and $99.8 \%$, respectively (Supplementary Fig. 2a-c). The sensitivities, specificities, and overall agreements of the RBD-specific $\lg A$, $\lg M$, or $\lg G$ kit and their combinations are also summarized in Supplementary Table 2. When combining the RBD $\lg A$ and $\lg G$ kits, the sensitivity, specificity, and overall agreement elevated to $99.1 \%, 100 \%$, and
$99.7 \%$, respectively. This is better than those when $\lg M$ and $\lg G$ kits are combined using our data or the previous data shown by others. $^{1-4}$

In order to investigate the seroconversion during COVID-19 pathogenesis, all the data from 216 sera samples were divided into six groups according to the time windows of collection after illness onset (Fig. 1d). At 4-10 days after symptom onset, the $\lg \mathrm{A}$ kit exhibited the highest positive diagnostic rate as $88.2 \%$ (15/17), while lgM and lgG kit showed detection rates of $76.4 \%$ (13/17) and $64.7 \%$ $(11 / 17)$, respectively. The 2 sera diagnosed as negative in the 4-10 days group by IgA kit were collected at the 4th day after illness onset, all other sera includes 2 at the 6 th day, 3 at the 7th day, 1 at the 8th day, 6 at the 9th day, and 3 at 10th day after illness onset were tested as positive. In the group of 11-41 days after symptom onset, both RBD $\lg A$ and $\lg G$ kit showed the same positive diagnostic rate of $99.5 \%$ (198/199). In contrast, lgM kit somehow showed a relatively lower positive diagnostic rate as $98.5 \%$ (196/199). These results suggest that including $\operatorname{lgA}$ in a test provides better diagnostic outcome in early stages. Overall, the medium seroconversion time for $\lg A$, $\lg M$, and $\lg G$ are 4-6, 4-6, and 5-10 days post symptom onset, respectively, if tested with the RBD-kits described in this study. While it generally follows a typical seroconversion and immunoglobulin class switching time course, our kits provides an early diagnosis solution due to high sensitivities.

To better understand the trends of antibody levels in all of the 87 COVID-19 patients (some of them contributed multiple samples), we plotted the median RLU reading according the time windows when sera were collected (Fig. 1e). IgA detection shows the highest sensitivity during about 4-25 days after illness onset. The median RLU of RBD-specific IgA reached the peak during 16-20 days after illness onset, and then began to decline but remained at relatively high reading until 31-41 days. The median RLU of RBD-specific IgG was the lowest in early disease stages but raised at 15 days post illness onset, the IgG reached its peak during 21-25 days after illness onset, and stayed at a relatively high reading until 31-41 days, suggesting that lgG is powerful for diagnostics at later stages. Although IgM reached its peak at early stages, the RLU reading was lower than that of $\lg A$ or $\operatorname{lgG}$.

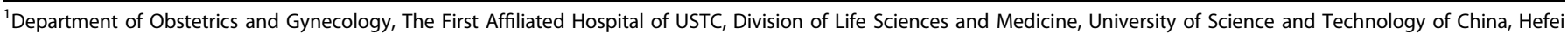

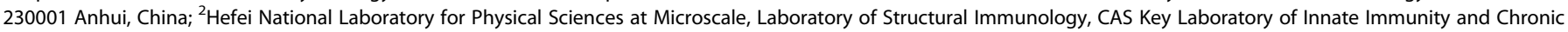
Disease, Division of Life Sciences and Medicine, University of Science and Technology of China, Hefei 230027 Anhui, China; ${ }^{3}$ Department of Infectious Diseases, The First Affiliated

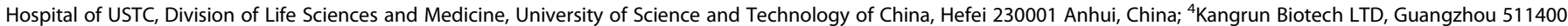

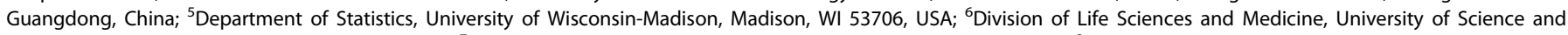
Technology of China, Hefei 230027 Anhui, China; ${ }^{7}$ Johns Hopkins University School of Medicine, Columbia, MD, USA; ${ }^{8}$ Department of Clinical Laboratory, The First Affiliated

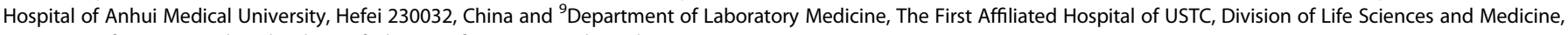
University of Science and Technology of China, Hefei 230001 Anhui, China

Correspondence: Yajuan Li (lyj106@ustc.edu.cn) or Xiaoling Ma (maxiaoling@ustc.edu.cn) or Tengchuan Jin (jint@ustc.edu.cn)
}

These authors contributed equally: Huan Ma, Weihong Zeng, Hongliang He

Received: 15 May 2020 Accepted: 18 May 2020

Published online: 28 May 2020 

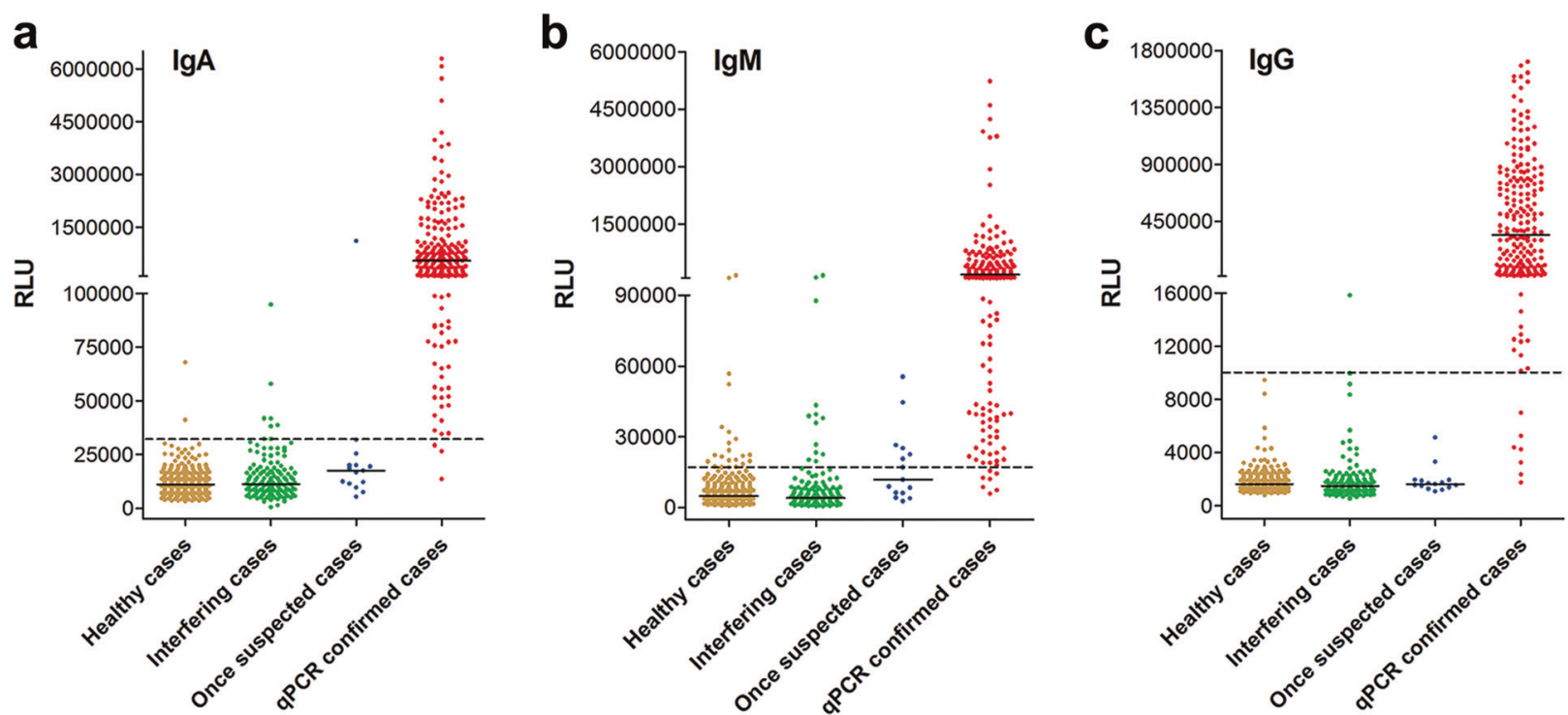

d

\begin{tabular}{|c|c|c|c|c|c|c|}
\hline \multirow{3}{*}{$\begin{array}{l}\text { Days after } \\
\text { illness onset }\end{array}$} & \multicolumn{6}{|c|}{ Positive samples diagnosed by RBD-specific antibodies } \\
\hline & \multicolumn{2}{|c|}{ IgA } & \multicolumn{2}{|c|}{ IgM } & \multicolumn{2}{|c|}{ IgG } \\
\hline & $\%$ & $\mathbf{n}$ & $\%$ & $\mathbf{n}$ & $\%$ & $\mathbf{n}$ \\
\hline $4-10$ & 88.24 & $15 / 17$ & 76.47 & $13 / 17$ & 64.71 & $11 / 17$ \\
\hline $11-15$ & 100 & $30 / 30$ & 100 & $30 / 30$ & 96.67 & $29 / 30$ \\
\hline $16-20$ & 100 & $55 / 55$ & 100 & $55 / 55$ & 100 & $55 / 55$ \\
\hline $21-25$ & 98.21 & $55 / 56$ & 100 & $56 / 56$ & 100 & $56 / 56$ \\
\hline $26-30$ & 100 & $35 / 35$ & 100 & $35 / 35$ & 100 & $35 / 35$ \\
\hline $31-41$ & 100 & $23 / 23$ & 86.96 & $20 / 23$ & 100 & $23 / 23$ \\
\hline
\end{tabular}
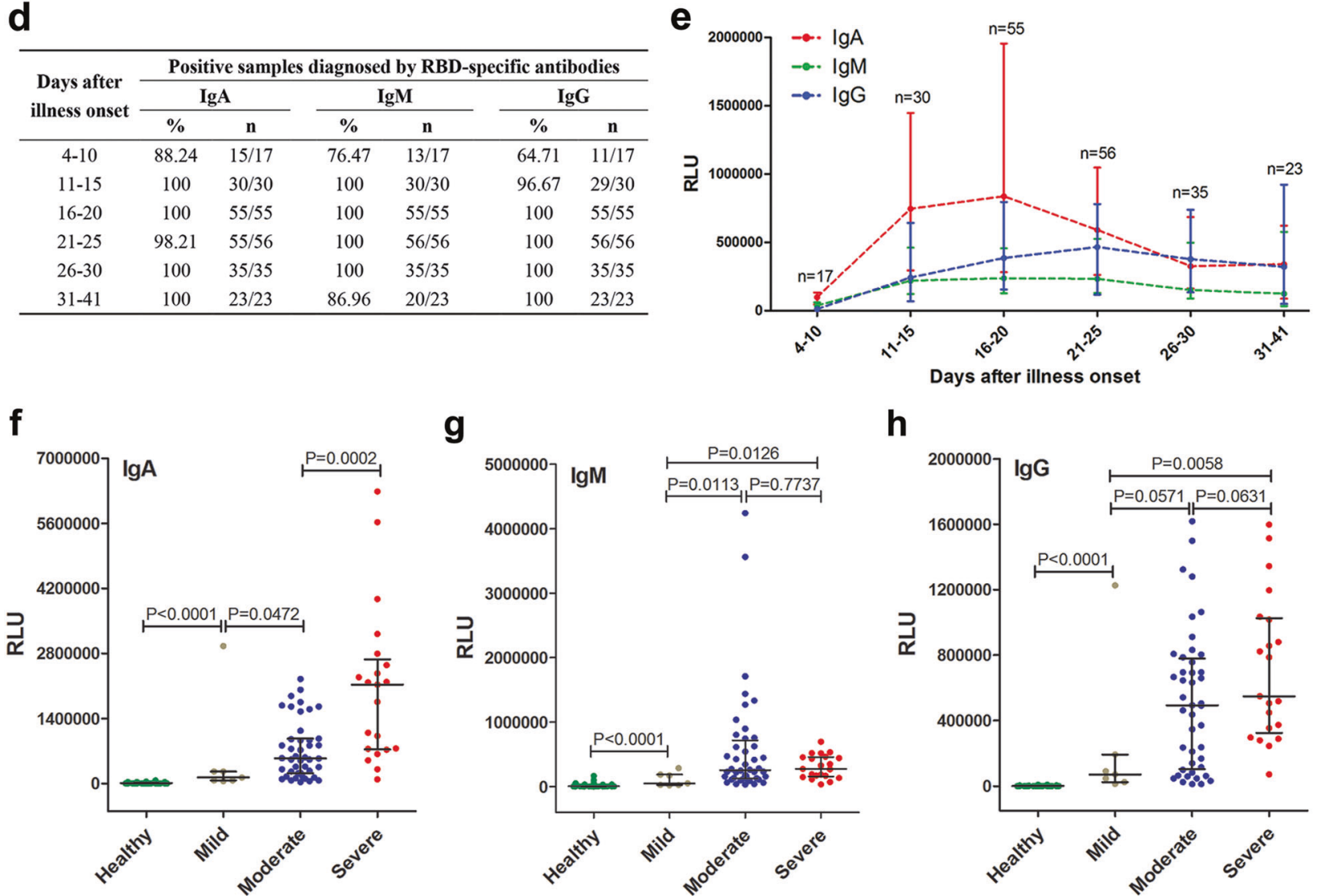

Fig. 1 Analysis of SARS-CoV-2 RBD-specific IgA, IgM, and IgG antibodies in 87 COVID-19 patients. Testing results of RBD-specific IgA (a), IgM (b), and IgG (c) kits using 330 healthy sera, 138 sera from other-type of patients who may interfere with the test, 15 sera of once-suspected pneumonia patients, and 216 sera of 87 qPCR-confirmed COVID-19 patients. RLU relative light units. Black bar indicates median values. The dotted line indicates the cut-off value for detecting of each isotypes of antibodies. $\mathbf{d}$ Sensitivity of RBD-specific $\operatorname{lgA}$, IgM, and IgG detection in serum samples obtained at different periods after illness onset. The kinetics of anti-RBD IgA, IgM, and IgG levels in sera of COVID-19 patients at different time windows was analyzed (e). The median values of RLU were plotted for each isotype of three antibodies. Bars indicate median with interquartile ranges. $\mathbf{f}-\mathbf{h}$ Serum antibody levels in healthy and three distinct severity groups of COVID-19 patients were analyzed. Healthy: 330 sera; Mild: 7 sera; Moderate: 44 sera; and Severe: 21 sera. The critically ill patients were included into the severe group. Only the data of serum antibody levels at 16-25 days after illness onset of COVID-19 patients were used 
We further divided the 87 patients into three severity groups based on established clinical classifications. Consistent with a previous report, ${ }^{7}$ we found that COVID-19 severity is correlated positively with age in our cohort (Supplementary Fig. 3). Patients with severe symptoms were significantly older (median age of 62.5) than those patients with moderate (median age of 46) and mild symptoms (median age of 30), as expected. We used the data of antibody levels at the period of 16-25 days after illness onset, when all of the three isotypes reached or were near their peaks (Fig. 1e). If there were more than one data points, the average value was taken. Serum IgM and lgG levels in moderate and severe COVID-19 patients were significantly higher than mild cases, while no significant difference was observed between severe and moderate patients (Fig. 1g, h). However, we found that IgA levels in severe cases were significantly higher than those mild or moderate cases (Fig. 1f). The molecular mechanism of this observation warrants future studies.

There are some limitations in this study at the current form. We used 216 serum samples from 87 confirmed COVID-19 patients in this study, and serum samples were not available every day for each patient. The earliest serum was collected at the 4th day, and last one was at the 41th day after self-reported illness onset. There are only 17 cases of serum samples collected within the first 10 days after illness onset; which consequently influenced the accuracy. Similarly, there were only 23 cases of serum samples taken after 30 days post illness onset, hampering an analysis of long-term antibody levels in recovered patients. We are currently following up some of the 87 convalescent COVID-19 patients who are willing to participate in further study. Nevertheless, this study provide valuable information regarding COVID-19 serological testing and seroconversion responses, especially for $\lg \mathrm{A}$ antibodies.

\section{ACKNOWLEDGEMENTS}

We acknowledge funding support from the Strategic Priority Research Program of the Chinese Academy of Sciences (XDB29030104), National Natural Science Foundation of China (Grant Nos.: 31870731 and U1732109), the Fundamental Research Funds for the Central Universities (WK2070000108), a COVID-19 special task grant supported by Chinese Academy of Science Clinical Research Hospital (Hefei) (YD2070002017 and YD2070002001), and the new medical science fund of USTC (WK2070000130)

\section{ADDITIONAL INFORMATION}

The online version of this article (https://doi.org/10.1038/s41423-020-0474-z) contains supplementary material.

Competing interests: Dehua Jiang is an employee of Kangrun Biotech LTD (Guangzhou, China). Tengchuan Jin, Huan Ma, Weihong Zeng in USTC and Dehua Jiang have applied a joining patent related to the antibody detecting kits. Other authors declare that they have no conflicts of interest.

\section{REFERENCES}

1. Zhang, W. et al. Molecular and serological investigation of 2019-nCoV infected patients: implication of multiple shedding routes. Emerg. Microbes Infect. 9, 386-389 (2020)

2. To, K. K.-W. et al. Temporal profiles of viral load in posterior oropharyngeal saliva samples and serum antibody responses during infection by SARS-CoV-2: an observational cohort study. Lancet Infect. Dis. 20, 565-574 (2020).

3. Zhao, J. et al. Antibody responses to SARS-CoV-2 in patients of novel coronavirus disease 2019. Clin. Infect. Dis. ciaa344. https://doi.org/10.1093/cid/ciaa344 (2020).

4. Li, Z. et al. Development and clinical application of a rapid lgM-lgG combined antibody test for SARS-CoV-2 infection diagnosis. J. Med. Virol. https://doi.org/ 10.1002/jmv.25727 (2020).

5. Guo, L. et al. Profiling early humoral response to diagnose novel coronavirus disease (COVID-19). Clin. Infect. Dis. ciaa310. https://doi.org/10.1093/cid/ciaa310 (2020).

6. Padoan, A. et al. IgA-Ab response to spike glycoprotein of SARS-CoV-2 in patients with COVID-19: a longitudinal study. Clin. Chim. Acta 507, 164-166 (2020).

7. Wolfel, R. et al. Virological assessment of hospitalized patients with COVID-2019. Nature. https://doi.org/10.1038/s41586-020-2196-x (2020).

\begin{abstract}
(c) (i)
Open Access This article is licensed under a Creative Commons Attribution 4.0 International License, which permits use, sharing, adaptation, distribution and reproduction in any medium or format, as long as you give appropriate credit to the original author(s) and the source, provide a link to the Creative Commons license, and indicate if changes were made. The images or other third party material in this article are included in the article's Creative Commons license, unless indicated otherwise in a credit line to the material. If material is not included in the article's Creative Commons license and your intended use is not permitted by statutory regulation or exceeds the permitted use, you will need to obtain permission directly from the copyright holder. To view a copy of this license, visit http://creativecommons. org/licenses/by/4.0/.
\end{abstract}

(c) The Author(s) 2020 